\title{
Strates
}

STRATES Matériaux pour la recherche en sciences sociales

$4 \mid 1989$

Dossier : images réfléchies. Paroles d'un paysan révolutionnaire

\section{Quelques réflexions sur les rapports de la géographie à l'image}

\section{Paul Chatelain}

\section{(2) OpenEdition}

\section{Journals}

Édition électronique

URL : http://journals.openedition.org/strates/4042

DOI : $10.4000 /$ strates.4042

ISSN : $1777-5442$

Éditeur

Laboratoire Ladyss

Édition imprimée

Date de publication : 31 décembre 1989

ISSN : 0768-8067

Référence électronique

Paul Chatelain, "Quelques réflexions sur les rapports de la géographie à l'image », Strates [En ligne], 4 I 1989, mis en ligne le 19 mai 2008, consulté le 08 septembre 2020. URL : http://

journals.openedition.org/strates/4042 ; DOI : https://doi.org/10.4000/strates.4042

Ce document a été généré automatiquement le 8 septembre 2020.

Tous droits réservés 


\title{
Quelques réflexions sur les rapports de la géographie à l'image ${ }^{1}$
}

\author{
Paul Chatelain
} ses dérivés. Le problème n'est pas là. Il est dans les conceptions qui semblent avoir prévalu dans les fondements iconographiques de la recherche. L'iconographie en cause recouvrant l'ensemble des images, que celles-ci soient utilisées comme matière première, comme support d'une démonstration, ou comme moyen de communication. La photo est, à cet égard, indissociable des compositions coloriées tirées de SPOT et de la gamme des cartes thématiques... Et bien sûr du discours sur l'espace...

7 A la base, il y a pour tous l'impératif de décrire des paysages et, d'une façon plus générale, de mettre en valeur ce qui est perceptible dans la pratique de l'espace par les groupes de la société.

Quelques constats sur la place de l'image dans la recherche

8 * Et d'abord, le dire a généralement tendance à remplacer le voir. Comme s'il y avait un a priori de la supériorité du discours sur l'image, ou simplement une antériorité 
reconnue de l'écrit sur d'autres façons d'appréhender le visible. Avec toujours le prétexte du manque de moyens techniques, c'est-à-dire financiers.

Il suffit d'invoquer l'illustration ou plutôt l'absence d'illustration des revues spécialisées dans la géographie!

Il est facile de renvoyer à des exemples d'écrivains de qualité... Il faut relire Dion ou le Tableau géographique de la France de Vidal de la Blache... Ou, pour rester dans l'actualité, suivre Julien Gracq dans ses descriptions de Nantes ou dans ses promenades dans Rome. L'auteur d'Autour des Sept Collines nous fournit les meilleures clefs de cette vision raisonnée du territoire, quand il dit, à propos de Rome, que là «Tout est alluvion et tout est allusion", quand il fait de la ville "une machine à remonter le temps" ou quand il souligne qu'elle "vacille entre ce qu'elle est et ce quelle signifie ».

* La vision géographique de l'espace privilégie, autant que le discours, la représentation cartographique du phénomène. Peu importe que la carte à thèmes reproduise une partie des trames qu'il importe de voir sur la photo (la morphologie agraire ou les tissus urbains), qu'elle rende visibles des inventaires de l'espace (le RGA, les formes du bâti) ou qu'elle transcrive des caractéristiques sur le contenu social et économique des lieux, qui, par définition, ne se voient pas directement. La carte est fondamentalement un substitut. Elle est là pour remédier aux imperfections d'une lecture immédiate qui pourrait être superficielle. On ne saurait nier que la conjonction du chiffre et de sa transcription cartographique soit, avec le discours, l'autre dimension forte de l'approche des territoires.

12 Bien sûr il faut tenir compte des servitudes d'échelle, de la focalisation des hypothèses ! et des contraintes de l'approche scientifique... Eliminons les faux débats. La carte est un acquis et un atout qu'il faut défendre. Et ses réussites dans la présentation des répartitions et des organisations sont innombrables! Notre interrogation se situe audelà. Elle part d'un constat de la rétention de l'image et n'est qu'un modeste «Pourquoi?»

13 Ne faut-il pas revenir à Bertin et à son Traité de Sémiologie Graphique? Rappeler que l'image, la photo sont, par définition, « polysémiques » en ce sens qu'elles ont la faculté d'avoir plusieurs significations et de se prêter à plusieurs interprétations. Sous cet angle, elles peuvent être le révélateur d'un système... Alors que la carte que l'on dit thématique ne peut être, pour l'essentiel, que «monosémique». Elle implique par le double jeu de la sélection des paramètres et de leur codification une orientation de la lecture... N'est-elle pas, à la limite, une façon de contourner la complexité des relations qui font l'intérêt des combinaisons spatiales?

$14{ }^{*}$ Lorsque l'image est systématiquement utilisée comme support technique de la recherche, c'est parce qu'elle fournit des renseignements dont la qualité d'expression est supérieure à la cartographie des chiffres.

15 Ainsi s'explique la réussite de la photo-interprétation depuis la thèse pionnière de P. Brunet sur l'originalité du paysage agraire au centre du Bassin parisien... Ou celle de l'exploitation des photos aériennes obliques... Le progrès actuel est dans la présentation des images satellites (Thematic Mapper, SPOT...) sur laquelle travaille, entre autre, l'équipe de J.P. Gilg aux Hautes Etudes. Mais la nature même de leur confection (il s'agit de la traduction colorée d'une sélection d'informations chiffrées) nous renvoie plus à la cartographie thématique qu'à la photo « polysémique ». 

des documents qui accompagnent le texte et ses cartes. Ou peuvent accompagner puisque les photos sont exceptionnelles et le plus souvent absentes des articles. Les revues spécialisées n'ont rien à voir avec GEO qui est une G.U. populaire. est-il plus commode d'etudier le contenu des images dans des ouvrages universitaires de vulgarisation scientifique. Certaines collections - «La France Région par Région ", publiée chez Larousse dans la décennie soixante-dix, l'"Atlas » et "Géographie de la France Moderne » lancé par Flammarion au début des années quatre-vingt - ont une qualité de photos qui répond à un souci de communication. Avec de superbes clichés de la «France par-dessus les toits» - on veut parler de ces photos aériennes obliques qui permettent de voir le paysage dans le champ de vision élargi au grand angle.

Létude critique de cette série d'ouvrages conduit cependant aux mêmes conclusions sur la marginalisation de l'image, même lorsque celle-ci semble se voir attribuer un rôle central.

Ne serait-ce que le fait que les vues sont sélectionnées a posteriori - après l'écriture du texte. Les clichés sont évidemment adaptés aux thèmes du chapitre. Mais ils ne sont jamais le support de ses paragraphes.

La sélection des illustrations est tout aussi révélatrice par la cohérence des choix que la signification des oublis. Cette sélection a été faite en privilégiant les représentations symboliques qui soulignent la spécificité des milieux naturels ou la richesse des patrimoines, qu'ils soient monumentaux (les centres-villes) ou de pratiques culturelles (la pétanque dans le Sud). Avec une volonté d'imposer l'image de marque d'un discours dominant sur le dynamisme régional (d'où la répétition des grands chantiers, de l'eau, de l'industrie, du tourisme ou de la rénovation urbaine).

21 Et, en corollaire, une sorte d'amnésie sur les scories de l'histoire, avec la mise entre parenthèses du dernier siècle d'urbanisation et d'industrialisation... Comme un désir d'exorciser les activités dépassées, les constructions anarchiques, le problème des pieds-noirs et celui des immigrés à propos des grands ensembles. Pour l'amateur de repères précis, citons, pour la France du Sud, l'étonnante image de la ZUP de la Paillade-Montpellier où l'idéalisation du jeu des volumes et des fausses couleurs fait oublier le reste (le contenant comme le contenu), ou cette image d'Epinal des agrosystèmes méditerranéens, où les oliviers centenaires, plus grecs que provençaux, font la nique au gel de $1956 \ldots$

Devant cette marginalisation de l'image, ou cette simple rétention de son usage, il n'est pas inutile de s'interroger. Ne serait-ce que parce que la recherche conditionne largement la pédagogie. Et que dans une société de la photo, de l'affiche et du film, on n'assure pas l'apprentissage critique du décodage.

Quelques ouvertures sur la pratique des autres

Il y a beaucoup à prendre dans la pratique des autres. Et plus encore dans la problématique qui sous-tend leur valorisation de ce mode d'expression qu'est l'image. Pour dépasser certains blocages et quelques appréhensions. En se persuadant qu'une alternative de la géographie est là.

* La rétention de l'image n'est-elle pas un refus du ponctuel et de l'événementiel comme moyen de prendre en compte ce qui est essentiel ? Est-il possible de généraliser à partir d'un instantané quand on se préoccupe de permanences et de structures? 


\section{formation, passé à l'architecture, une des rares disciplines qui intègrent} systématiquement dans le cursus pédagogique l'initiation critique au décryptage des paysages :

32 "Le paysage en tant qu'objet ne renvoie pas à lui-même, mais au sujet qui l'appréhende et aux structures économiques et sociales, qui lui ont donné naissance, contribuent à l'entretenir (paysage, fonctionnel), à la modifier (dynamique progressive et régressive) et à l'abandonner (paysage fossile) « ... «le paysage est signé. Il porte la marque des systèmes économiques qui l'ont engendré" ... «Il est toujours entre permanence et devenir $»^{2}$.

Un élément de réponse se trouve sans doute dans cette volonté qu'ont eu les grands photographes de témoigner en archivistes qui s'engagent socialement. Les références ne manquent pas dans les attendus d'expositions, les simples légendes de photos ou la philosophie des Mémoires.

C'est Brassai qui dit en 1971 (Le Mois de la Photo, Carnavalet 1988) :

" Je dois admettre que j'ai toujours fait du reportage, mais un reportage en profondeur qui décrivait la ville et l'époque à laquelle je vivais... Ce ne sont pas les sociologues qui pénètrent les choses, mais les photographes qui sont des observateurs au cœur même de notre temps" . Et le même : «Le monde n'a de signification que dans l'instant ». est exposée dans un catalogue de la même série d'expositions du Mois de la Photo (cours Bel Air, Paris XII ${ }^{e}$ ) - rejoint presque terme à terme la conscience des reporters du Paris des années cinquante (Doisneau, Izis, Brassai, Cartier-Bresson...). Dans un pays où la photo est encore une sous-culture, on retrouve le même impératif de témoigner sur la signification de l'état et de la pratique des lieux... avec l'idée qu'il y a une continuité dans les modes d'expression qui fait que la photo d'aujourd'hui s'inscrit dans la tradition de Tourgueniev et de la littérature du dernier siècle...

série qui s'intitule "Les cendrillons de la grande ville»: «De la vie des jeunes filles venues de la Province à Moscou pour travailler sur annonces » (1987).

essentielles que sontlenracin leur utilisation?

Rassurons-nous, les strates que l'on peut identifier renvoient bien à des structures qui s'inscrivent dans une histoire et sont interdépendantes dans leur signification actuelle.

* Et peut-être surtout la sélection de l'image n'est jamais neutre. Elle est toujours une façon de s'engager. A la limite, de se démarquer de l'iconographie officielle qui reproduit un type de discours dominant sur les états du territoire.

C'est aussi une façon de communiquer avec un type de lecteurs; on pourrait aussi bien dire, en termes de marketing, de cibler une clientèle. "Je ne travaille pas sur le tableau ", disait Matisse, "mais sur le spectateur ». Or la parcellisation des thèmes de recherche conduit à l'individualisation des publics et donc au choix de modes d'expression de plus en plus spécialisés. Et cela au détriment de l'image quand celle-ci devient un outil banal et universel de communication. On parle ici d'image au sens d'image de marque, de message qu'il faut faire passer. Une autre image, objet de contrats et sujet d'études critiques... Le logo des entreprises, la publicité des villes et des régions, le cliché des 
agences de voyage, la société des affiches électorales... Tout un champ d'études que l'on commence à prospecter, et sur lequel il y a beaucoup à apprendre des agences de publicité.

Si lire, c'est aujourd'hui voir, apprendre à lire, c'est aujourd'hui apprendre à voir. L'anesthésie de la vision n'est pas seulement un problème de pédagogie. La discipline qui a le plus vocation à décoder l'espace n'a-t-elle pas tendance, dans ses recherches, à exorciser l'image?

\section{NOTES}

1. A propos du Mois de la Photo, Paris, décembre 1988.

2. «Lire les paysages », Colloque de Saint Etienne, 24 et 25 novembre 1983.

\section{RÉSUMÉS}

Quelques réflexions sur les rapports de la géographie à l'image.

La recherche géographique n'a-t-elle pas tendance à exorciser l'image?

Some thoughts on the relationships between geography and images.

Is there a tendency to exorcize images in geographical research?

INDEX

Mots-clés : anesthésie de la vision

Keywords : anaesthesia of the vision

\section{AUTEUR}

\section{PAUL CHATELAIN}

Maître de conférences à Paris-I, il a publié avec Xavier Browacys Les France du travail, PUF, 1985. 